\title{
Skin photoage chemoprotection effect of Moutan Cortex through
}

\section{regulation of antimelanogenic pathway}

\author{
Leong-Perng Chan a,b, Ya-Ping Tseng ${ }^{\mathrm{c}}$, Tzung-Han Chou ${ }^{\mathrm{d}}$, Hsiou-Yu Ding ${ }^{\mathrm{e}}$, Da-Long Cheng ${ }^{\mathrm{f}}$, Wang Yaying g, \\ Guey-Horng Wang h,*, Chia-Hua Liang e,* \\ a Institute of Clinical Medicine, Kaohsiung Medical University, Kaohsiung, Taiwan \\ ${ }^{\mathrm{b}}$ Department of Otolaryngology-Head and Neck Surgery, Kaohsiung Medical University Hospital, Kaohsiung Medical \\ University, Kaohsiung, Taiwan \\ c Institute of Basic Medical Sciences, National Cheng Kung University, Tainan, Taiwan \\ ${ }^{d}$ Department of Chemical and Materials Engineering, National Yunlin University of Science and Technology, Yunlin, \\ Taiwan \\ ${ }^{\mathrm{e}}$ Department of Cosmetic Science, Chia Nan University of Pharmacy and Science, Tainan, Taiwan; \\ ${ }^{\mathrm{f}}$ Department of Computer and Communication, Shu-Te University, Kaohsiung, Taiwan \\ ${ }^{g}$ Research Center of Natural Cosmeceuticals Engineering (RCNCE), Xiamen Medical College, China \\ ${ }^{\mathrm{h}}$ Fujian Key Laboratory on Biological Engineering for Medicinal Materials, Xiamen Medical College, China
}

*Corresponding Author: 2659294971@qq.com; tinna_ling@mail.cnu.edu.tw

\begin{abstract}
Ultraviolet is a major factor to cause the DNA damage. Erythema, edema, and pigmentation are observed in the acute inflammation, whereas photo-ageing and skin cancers are found in the chronic one. Moutan Cortex is a traditional Chinese herbal medicine. Paeoniflorin and oxypaeoniflorin are major active compounds among the extracts of Moutan Cortex. The effects of paeoniflorin and oxypaeoniflorin against pigmentation, tyrosinase activation and expression of melanogenesis related genes and proteins in B16 cells were investigated. In $\mathrm{Hs} 68$ and B16 cells treated with paeoniflorin and oxypaeoniflorin did not show apparent cytotoxicity compared to ascorbic acid. Paeoniflorin and oxypaeoniflorin inhibited mushroom tyrosinase activity, reduced cellular tyrosinase, DOPA oxidase activity and melanin content in B16 cells. Western blotting analysis showed that treatment with paeoniflorin and oxypaeoniflorin for $72 \mathrm{~h}$ down-expression the levels of melanocortin-1 receptor (MC1R), mecrophthalmia-associated transcription factor (MITF), tyrosinase, tyrosinase-related proteins-1 (TRP-1) and TRP-2. These results suggested that paeoniflorin and oxypaeoniflorin have antimelanogenesis activities, including inhibition of melanogenic enzymes and down-regulating the melanogenesis related proteins. Moreover, these two compounds are safety and provide a new device for skin whitening and abnormal pigmentation
\end{abstract}

disease.

Keywords: Moutan Cortex, melanogenesis, photoage chemoprotection.

\section{Introduction}

Melanin is the main component determining the color of skin. The major role of melanin is to protect the skin from damaging effects of ultraviolet. Ultraviolet is a major factor to cause the DNA damage. Erythema, edema, and pigmentation are observed in the acute inflammation, wherease photo-ageing and skin cancers are found in the chronic one $(1,2)$.

In mammals, melanin is synthesized in the melanosomes of melanocytes by binding $\alpha$-melanocyte-stimulating hormone $(\alpha-\mathrm{MSH})$ to $\mathrm{MC1R}$, which increases the available cyclic AMP and boots the subsequent activation of MITF. The MITF up-regulates the expression of melanogenic enzymes including tyrosinase, TRP-1/5,6-dihydroxyindol-2-carboxylic acid oxidase and TRP-2/DOPAchrome tautomerase (3).

Melanin biosynthesis inhibitors are not only powerful skin-whitening agents in cosmetics but are also potent remedies for heterogeneous pigmentation. Various agents have been identified as effective in reducing melanogenesis. They include arbutin, hydroquinone and kojic acid, which are reportedly tyrosinase inhibitors. Additionally, although numerous tyrosinase inhibitors have been utilized 
extensively to lighten skin, they have several side effects, such as the adverse cutaneous toxicity of hydroquinone and the tumor-promoting effect of kojic acid (4).

Moutan Cortex is used as an analgesic, sedative, antipyretic, anti-inflammatory and anti-atherosclerosis agent in traditional Chinese medicine. Paeoniflorin and oxypaeoniflorin are major active compounds among the extracts of Moutan Cortex (5). The antimelanogenesis behaviors of paeoniflorin and oxypaeoniflorin from Moutan Cortex are not completely understood. Accordingly, this study evaluated the antimelanogenesis effects of paeoniflorin and oxypaeoniflorin.

\section{Results}

\subsection{Cell Viability Assay}

Cells $\left(1 \times 10^{5}\right.$ cells $\left./ \mathrm{ml}\right)$ were plated in $100 \mu \mathrm{l}$ of 96-well multidishes and treated with serial concentrations of paeoniflorin, oxypaeoniflorin and ascorbic acid for $72 \mathrm{~h}$. Agents were dissolved at a concentration of $100 \mathrm{mg} / \mathrm{ml}$ in dimethylsulfoxide (DMSO) stock solution. The control groups were treated with DMSO, and the final DMSO concentration did not exceed $0.1 \%$. The cell viability was measured by the MTT [3-(4,5-dimethyl-thiazol-2-yl)-2,5-diphenyl-tetrazolium bromide] assay.

The survival rates of murine melanoma B16 cells, and human fibroblast Hs68 cells that were exposed to paeoniflorin and oxypaeoniflorin were compared to those exposed to ascorbic acid using MTT assay. As shown in Fig. 1 , paeoniflorin and oxypaeoniflorin did not exhibit any appreciable cytotoxic activity at a dose of $20 \mu \mathrm{M}$.

\subsection{Effect of Paeoniflorin and Oxypaeoniflorin on Tyrosinase activity}

Paeoniflorin, oxypaeoniflorin and ascorbic acid activities of mushroom tyrosinase were determined with L-DOPA, respectively as the substrate by measuring the rate of DOPAchrome formation at $475 \mathrm{~nm}$.

The effects of paeoniflorin and oxypaeoniflorin on the oxidation of L-DOPA catalyzed by mushroom tyrosinase were first studied. The results demonstrated that the activity of mushroom tyrosinase was inhibited by paeoniflorin, oxypaeoniflorin and ascorbic acid in a concentration-dependent manner (Fig. 2). Paeoniflorin and oxypaeoniflorin showed moderate inhibitory effect on mushroom tyrosinase activity, whereas ascorbic acid had a strong inhibitory effect.

\subsection{Effect of Paeoniflorin and Oxypaeoniflorin on Synthesis of Melanin}

B16 cells $\left(1 \times 10^{5}\right.$ cells $\left./ \mathrm{ml}\right)$ were seeded in $3 \mathrm{ml}$ of 6-well plates for 24 h. $\alpha$-MSH $(1 \mu \mathrm{M})$ was then added and cells were treated with serial concentrations of paeoniflorin, oxypaeoniflorin and ascorbic acid in phenol red free DMEM for $72 \mathrm{~h}$. One hundred $\mu \mathrm{l}$ aliquots of media were then placed in 96-well plates and optical densities were measured at $405 \mathrm{~nm}$. Cells were then scraped from the plates and resuspended in cell culture media containing trypan blue (final concentration $0.1 \% \mathrm{w} / \mathrm{v}$ ) and counted using a haemocytometer. Mean increases in extracellular melanin production per cell were expressed as a percentage of basal melanin production in control cells.

In identifying the mechanism of the synthesis of melanin, the effects of paeoniflorin and oxypaeoniflorin on tyrosinase activity, on the synthesis of tyrosinase upon the stimulation of B16 cells with $\alpha-\mathrm{MSH}$, and on the depigmentation of melanin were evaluated. Paeoniflorin and oxypaeoniflorin inhibited melanin formation more strongly that did ascorbic acid (Fig. 3).

\subsection{Effect of Paeoniflorin and Oxypaeoniflorin on Melanogenesis-Related Signaling Pathway}

Changes of melanin synthesis-related proteins, including MC1R, MITF, tyrosinase, TRP-2, and TRP-1 expressions in B16 cells $\left(1 \times 10^{5}\right.$ cells $\left./ \mathrm{ml}\right)$, upon treatment with $\alpha$-MSH $(1 \mu \mathrm{M})$ alone or with $\alpha$-MSH plus $20 \mu \mathrm{M}$ concentration of paeoniflorin and oxypaeoniflorin for $72 \mathrm{~h}$, were evaluated by western blotting. The cells were lysed in NP40 lysis buffer $\left[\begin{array}{lll}50 & \mathrm{mM}\end{array}\right.$ 4-(2-hydroxyethyl)-1-piperazineethanesulfonic acid (HEPES) (pH 7.25), $150 \mathrm{mM} \mathrm{NaCl}, 50 \mu \mathrm{M} \mathrm{NaF}, 2 \mathrm{mM}$ ethylenediaminetetraacetic acid (EDTA), $1 \mathrm{mM}$ sodium vanadate, $1.0 \% \mathrm{NP} 40$ and $2 \mathrm{mM}$ phenylmethylsulfonyl fluoride (PMSF)]. The total cell lysate was centrifuged at $12000 \mathrm{rpm}$ for $5 \mathrm{~min}$, and protein concentration was determined by an ESL protein assay (Boehinger-Mannheim) with bovine serum albumin as standard. Cell lysate protein $(40 \mu \mathrm{g})$ was subjected to $12 \%$ sodium dodecyl sulfate polyacrylamide gel electrophoresis and electrophoretically transferred to nitrocellulose membranes. The membranes 


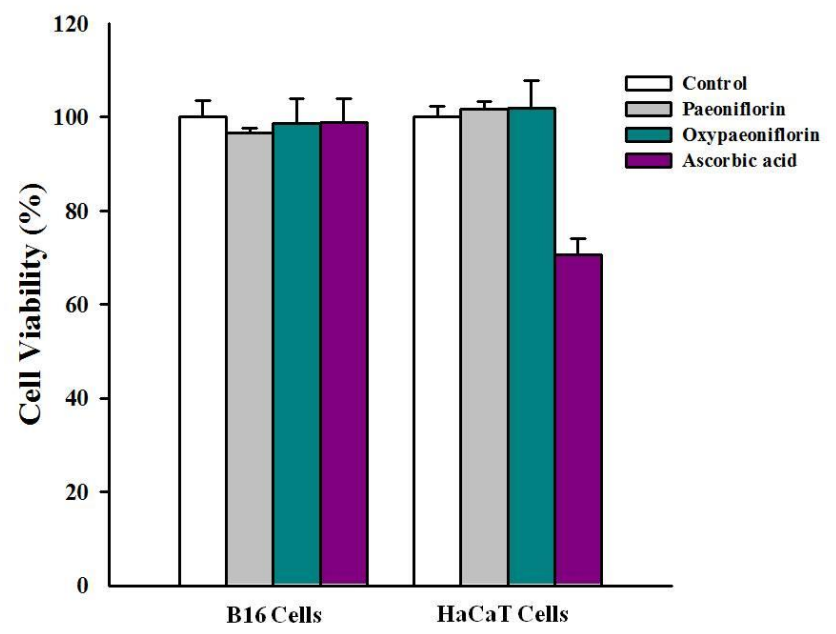

Fig. 1. Effects of paeoniflorin, oxypaeoniflorin and ascorbic acid on viability of B16 and HaCaT cells

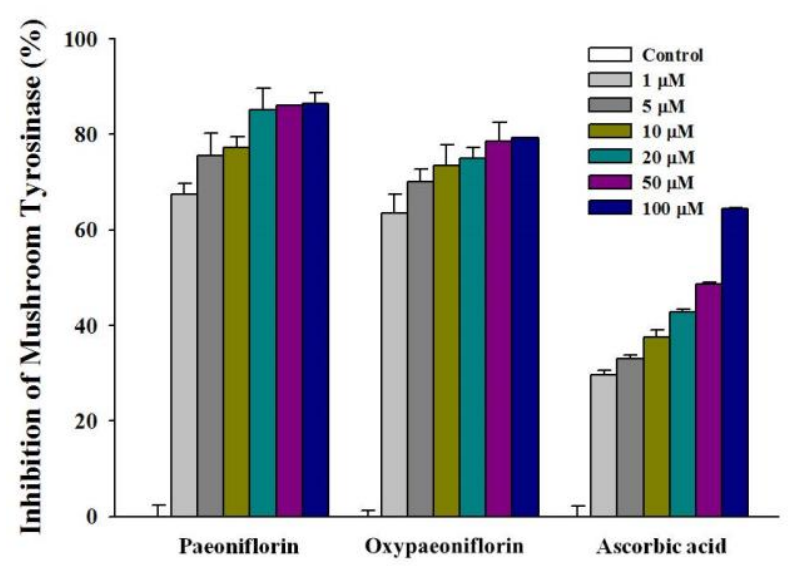

Fig. 2. Inhibition of paeoniflorin, oxypaeoniflorin and ascorbic acid on mushroom tyrosinase activity

were blocked in 5\% skim milk. Blots were incubated with the antibodies against MC1R, MITF, tyrosinase, TRP-1, TRP-2 and $\beta$-actin (Santa Cruz Biotechnology, Santa Cruz, CA, U.S.A.). The membranes were incubated with the appropriate secondary antibody conjugated with horseradish peroxidase (Bio-Rad, Hercules, CA, U.S.A.). Blots were visualized on X-ray film with enhanced ECL blotting detection reagents (Amersham, Piscataway, NJ, U.S.A.).

To investigate whether paeoniflorin and oxypaeoniflorin influences the expression of melanogenesis-related genes and proteins, including MC1R, MITF, tyrosinase, TRP-2 and TRP-1, the protein levels in B16 cells were measured using western blot analysis after treatment with $20 \mu \mathrm{M}$ of paeoniflorin and oxypaeoniflorin. As shown in Fig. 4, down-regulation of MC1R, MITF,

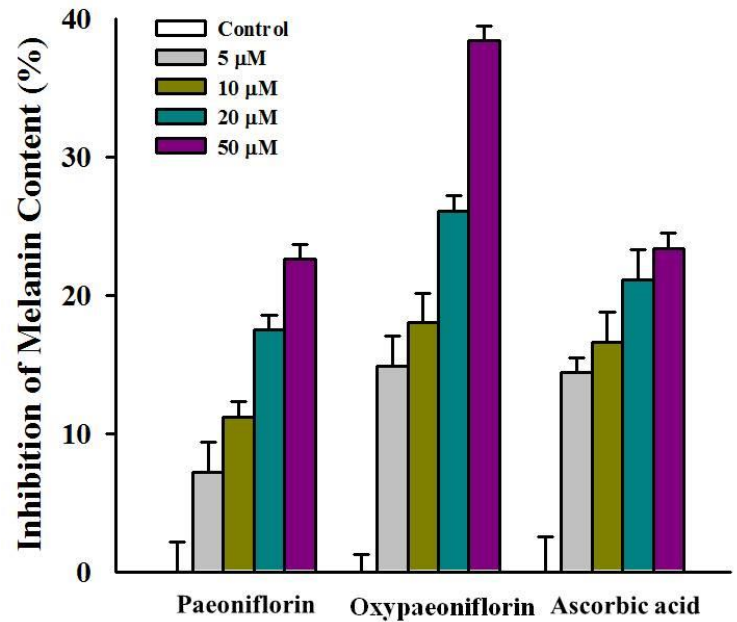

Fig. 3. Inhibition of melanin synthesis in B16 cells by paeoniflorin and oxypaeoniflorin.

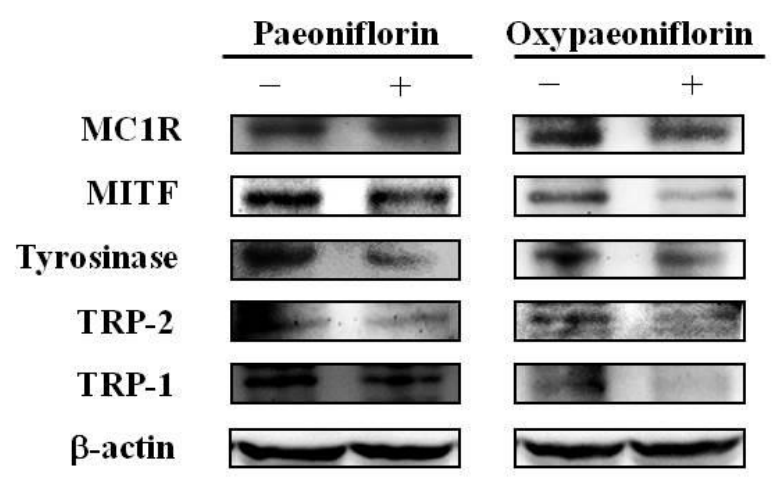

Fig. 4. Expressions of proteins that encode MC1R, MITF, tyrosinase, TRP-2, and TRP-1 in paeoniflorin and oxypaeoniflorin-treated B16 cells.

tyrosinase, TRP-2 and TRP-1 protein expression by paeoniflorin and oxypaeoniflorin in B16 cells for $72 \mathrm{~h}$. These results indicate that the suppression by paeoniflorin and oxypaeoniflorin of hypopigmentation is linked to the down-regulation of melanogenesis-related signaling pathways.

\section{Copyright}

This study were original and unpublished researches in other conferences and journals.

\section{Conclusions}

The study demonstrated the antimelanogenesis properties of paeoniflorin and oxypaeoniflorin from 
Moutan Cortex in detail. The non-toxic paeoniflorin and oxypaeoniflorin significantly reduced cellular tyrosinase activity, DOPAquinone content, and melanin formation in B16 cells. Treated with paeoniflorin and oxypaeoniflorin may have resulted from down-expression of MC1R, MITF. Paeoniflorin and oxypaeoniflorin have many potential applications in cosmetics and foods.

\section{Acknowledgment}

The authors would like to thank the Ministry of Science and Technology of the Republic of China, Taiwan, for financially supporting this research under Contracts No. MOST 103-2320-B-041-001-MY3.

\section{References}

(1) Bruce K Armstrong and Anne Kricker: "The epidemiology of UV induced skin cancer", Journal of Photochemistry and Photobiology B: Biology. Vol. 63, No. 1-3, pp 8-18, 2001.

(2) M. Ichihashi, M. Ueda, A. Budiyanto, T. Bito, M. Oka, M. Fukunaga, K. Tsuru, T. Horikawa: "UV-induced skin damage", Toxicology. Vol. 189, No. 1-2, pp. 21-39, 2003

(3) Yi-Pei Lin, Feng-Lin Hsu, Chien-Shu Chen, Ji-Wang Chern, Mei-Hsien Lee: "Constituents from the Formosan apple reduce tyrosinase activity in human epidermal melanocytes", Phytochemistry. Vol. 68, No. 8, pp. 1189-99, 2007

(4) Kwang Seok Ahn, Ki-Young Moon, Jinseon Lee, Yeong Shik Kim: "Downregulation of NF-kappaB activation in human keratinocytes by melanogenic inhibitors", Journal of Dermatological Science. Vol. 31, No. 3, pp. 193-201, 2003

(5) Rie Furuya, Honghai $\mathrm{Hu}$, Zhenya Zhang and Hideyuki Shigemori: "Suffruyabiosides A and B, two new monoterpene diglycosides from moutan cortex", Molecules. Vol. 17, No. 5, pp. 4915-23, 2012 